\title{
Looking Deep from the South Pole: Star Formation in the Thermal Infrared
}

\author{
Michael G. Burton, John W.V.Storey and Michael C.B. Ashley \\ Joint Australian Centre for Astrophysical Research in Antarctica, \\ School of Physics, University of New South Wales, Sydney, NSW 2052, Australia
}

\begin{abstract}
. ${ }^{1}$
The Antarctic Plateau provides the pre-eminent conditions on the Earth for widefield imaging at thermal infrared wavelengths. We describe a project to equip the $60 \mathrm{~cm}$ SPIREX telescope at the South Pole with a large format $(1024 \times 1024)$ IR array camera (Abu) to demonstrate this potential. With it we aim to survey the Large Magallenic Cloud for sites of massive star formation at $3.5 \mu \mathrm{m}$ at the 1.4 " diffraction limit of the telescope. We also discuss the potential for studying extra-galactic star formation through a deep survey of the Hubble Deep Field-South in this band. We compare the sensitivity of such surveys from Antarctica with those from mid-latitude sites, and propose a 2 m-class telescope, SPIRIT, which would be able to achieve unique new science at low cost compared to the new generation of $8 \mathrm{~m}$-class telescopes now under construction.
\end{abstract}

\section{Introduction}

Understanding star formation is an active research area in contemporary astrophysics. Of interest is the study of processes which occur as a single star forms, their collective effects during group and cluster formation, and the birth of entire galaxies. We seek to understand the dependence of these events on the local environment of the molecular clouds in which they occur. This endeavour is very much the realm of infrared and millimetre astronomy, both to peer into the murky depths of the clouds to see the young stars, and to measure the properties of the natal gas from which they came. A key goal of these studies is to undertake a complete population census of regions of star formation in order to determine the number and types of stars that form in them, and how this varies between different complexes. For this, observations in the thermal infrared $(\lambda>3 \mu \mathrm{m})$ are necessary. These wavelengths not only penetrate to the depths of cloud cores, but also allow us to distinguish between the embedded population and background stars. In simple terms, young stellar objects are surrounded by warm (few hundred K) disks which emit strongly at $\lambda>3 \mu \mathrm{m}$, and thus are readily distinguished in infrared colour-colour diagrams (e.g. $[1.65 \mu \mathrm{m}-2.2 \mu \mathrm{m}] /[2.2 \mu \mathrm{m}-3.8 \mu \mathrm{m}]$ ) from reddened stars (e.g. see Figure 1). Near-IR colour-colour diagrams (e.g. $[1.25 \mu \mathrm{m}-1.65 \mu \mathrm{m}] /[1.65 \mu \mathrm{m}-2.2 \mu \mathrm{m}])$,

\footnotetext{
${ }^{1}$ Paper presented at ESO/ATNF workshop 'Looking Deep in the Southern Sky', Sydney, Australia, 10-12 December, 1997. To appear in the 'ESO workshop proceedings' series, editors R. Morganti $\& W$. Couch.
} 
while relatively easy to construct due to the better sensitivities available, show only small IR excesses due to disks. These are readily confused with reddening vectors, and also fail to include the most deeply embedded sources.
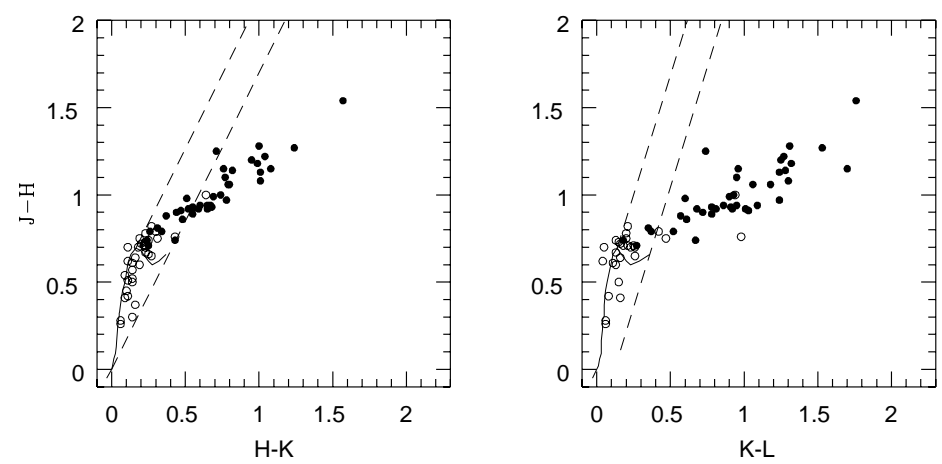

Fig. 1. Near-IR colour-colour diagrams for the nearby Taurus-Auriga molecular cloud (Strom et al. 1989). Left, J-H as a function of $\mathrm{H}-\mathrm{K}$; right, $\mathrm{J}-\mathrm{H}$ as a function of $\mathrm{K}-\mathrm{L}$. Filled circles represent pre-main sequence stars with spectroscopic evidence for accretion disks; open circles represent stars without. In both diagrams, the solid line corresponds to the main sequence and the dotted lines denote the reddening band. It is readily apparent how much clearer the separation between stars with and without disks is using the $\mathrm{K}-\mathrm{L}$ colour index compared to the $\mathrm{H}-\mathrm{K}$ colour $(\mathrm{J}=1.25 \mu \mathrm{m}, \mathrm{H}=1.65 \mu \mathrm{m}$, $\mathrm{K}=2.2 \mu \mathrm{m}$ and $\mathrm{L}=3.6 \mu \mathrm{m}$ ).

The problem has been that at $3.8 \mu \mathrm{m}$ sensitivities are typically $4-5$ magnitudes worse than at $2.2 \mu \mathrm{m}$ from most observing sites, thus limiting the work that has been done at this wavelength. Needed are deep, wide-field surveys of comparable sensitivity to those conducted at $2.2 \mu \mathrm{m}$ in order to determine the complete stellar membership of a star formation region. Such an opportunity is afforded a telescope on the Antarctic Plateau in view of the vastly reduced thermal background at these wavelengths over temperate sites. In this paper we describe a project now underway at the South Pole which aims to demonstrate that such surveys are feasible, with an initial objective being a $3.8 \mu \mathrm{m}$ survey of the Large Magallenic Cloud for massive star formation.

\section{The Antarctic Plateau}

The Antarctic plateau provides unique conditions on the Earth for the conduct of observational astronomy. The air is thin, dry and cold and the weather stable, attributes all offering gains to the observational astronomer. These conditions are quite different to those experienced at Antarctic coastal locations, which are frequently subject to violent storms. 
The plateau is over $3,000 \mathrm{~m}$ in elevation, rising to over $4,000 \mathrm{~m}$ at Dome $\mathrm{A}$. An average year-round temperature of $-50^{\circ} \mathrm{C}$, falling to $-90^{\circ} \mathrm{C}$ at times, vastly reduces the thermal background in the near-IR. The precipitable water vapour content of the air is typically around $250 \mu \mathrm{m}$ and can fall below $100 \mu \mathrm{m}$, opening up new windows in the infrared and sub-millimetre regimes to ground-based observation. The lack of a diurnal temperature cycle and the low wind speeds on the highest parts of the Antarctic plateau provide conditions of extraordinarily stability, benefiting a wide range of observational programs (Burton et al. 1994).

In the near-IR, from 2.2 to $5 \mu \mathrm{m}$, the thermal emission from sky and telescope at the South Pole has been measured to be 20-100 times less than at Mauna Kea (Ashley et al. 1996, Nguyen et al. 1996). However, while the greatest gains occur at $2.4 \mu \mathrm{m}$, it is only in a relatively narrow $(0.2 \mu \mathrm{m}$ wide) window. The entire L-band (3-4 $\mathrm{m}$ ) experiences a background reduction of 20-40 times that of Mauna Kea, opening up the possibility of deep observations in the thermal IR that simply cannot be conducted from any other ground based site. In the mid-IR measurements also show reductions of up to 20 in the background, a result of a reduced atmospheric emissivity in addition to the temperature drop. There is also exceptional stability in the DC level of the sky (Smith \& Harper, 1997).

The stable atmospheric conditions, tenuous air and absence of jet streams combine to produce conditions of superb clarity, or 'super-seeing'. Mitigating against these positive attributes, however, is the presence of a strong inversion layer which occurs on the most stable days of winter, when the air temperature can rise by $10^{\circ} \mathrm{C}$ in a few metres. This produces relatively poor ice-level seeing, $1.5^{\prime \prime}$. However it occurs almost entirely in the lowest $\sim 100 \mathrm{~m}$ of the atmosphere; there is virtually no contribution from above this height (Marks et al. 1996, 1998). The seeing is thus of a quite different nature to that encountered at temperate latitudes, with a much larger isoplanatic angle and coherence time for the seeing cells. The depth of the inversion layer decreases with elevation on the Plateau, and on top of the ice Domes may be small enough that a telescope on a raised platform will be above it entirely.

The US NSF has funded the Center for Astrophysical Research in Antarctica (CARA) to establish an astronomical observatory at the Amundsen-Scott South Pole station. Building upon a series of isolated experiments, construction of the observatory is now well advanced. Three major experiments have wintered over, an infrared camera (on the 60-cm SPIREX telescope), a sub-millimetre spectrometer (the 1.7-m AST/RO telescope), and a microwave background anisotropy experiment (COBRA). An extensive site-testing program has been conducted, with plans to extend it to the high points of the plateau, Domes A and C, using an automated observatory (the 'AASTO', Storey 1998).

\section{SPIREX/Abu}

SPIREX, the 'South Pole InfraRed EXplorer', is a 60-cm telescope built in 1994 by CARA in order to conduct observations from $2.4 \mu \mathrm{m}$ from the South Pole to 
determine whether the postulated 'cosmological window' on the Universe exists (Harper 1989, Hereld 1994). The window indeed existed, but the background turned out to be $\sim 5$ times higher than anticipated (though still some 40 times better than at temperate sites). However, as a result of parallel measurements using the IRPS (Ashley et al. 1996), the original IR spectrometer of the AAT, it was demonstrated that the entire $\mathrm{L}-$ band $(3-4 \mu \mathrm{m})$ has even greater potential as a new window, especially while only small aperture telescopes are available at the site.

An agreement between the Universities of Chicago and Ohio State, NASA Goddard, the US National Optical Astronomy Observatory and the University of New South Wales, has resulted in a project to upgrade the SPIREX telescope and install a state-of-the-art IR focal plane array; NOAO's "Abu" camera, incorporating a $1024 \times 1024 \mathrm{InSb}$ Aladdin array, with broad band and line imaging filters. UNSW's contribution includes the addition of a CCD fast guider and a tip-tilt secondary mirror, to improve the tracking and pointing of SPIREX. Automated observing and reduction protocols have been designed by Ohio State to allow continuous observing when conditions permit. This upgrade has taken place during the 1997/98 summer season at the Pole, with Australian participation funded by the Major National Research Facilities Program.

The system has been optimised for the thermal infrared L-band, around $3.5 \mu \mathrm{m}$. Expected sensitivities are given in Table 1 through the 6 filters. These include both summer and winter time figures as in the thermal IR day time operation will be possible with only slightly reduced sensitivities. Abu has an $11^{\prime}$ circular field of view with 0.7 " pixel scale, and will fully-sample at the diffraction limit of 1.4". With a Fried parameter of $\sim 50 \mathrm{~cm}$ at $3.5 \mu \mathrm{m}$, tip-tilt optics should allow us to obtain near-diffraction limited images even in poor seeing conditions.

\section{Plans}

There are many projects that SPIREX/Abu will be well suited to. These include:

- Line imaging of embedded HII regions along the southern galactic plane in the $\mathrm{Br} \alpha$, PAHs and $\mathrm{H}_{2}$ filters, to study the environment of the ionized, neutral and molecular mediums of massive star forming regions. Of particular interest is the NGC 6334 region.

- A survey of the Large Magallenic Cloud for massive star formation at L-band $(3.5 \mu \mathrm{m})$. Spending 1 hour per position, a $2^{\circ} \times 2^{\circ}$ region can be fully-sampled in a month of telescope time. Several thousand stars should be visible down to $\mathrm{L}=15.5$ magnitudes. While this only allows us to sample the top end of the mass distribution, it will now be possible to survey the LMC in a systematic manner for the regions where these stars form. This will be the first in-depth study of extra-galactic star formation, which is of interest in its own right. However studying star formation in the LMC, occuring in a lower metal abundance environment than our Galaxy, will also yield invaluable insights into how primordial star formation proceeds, as it presumably takes place in near-metal-free environment. 
Table 1. Calculated Sensitivity of SPIREX/Abu at the South Pole

\begin{tabular}{|c|c|c|c|c|c|c|}
\hline $\begin{array}{l}\text { Wavelength } \\
(\mu \mathrm{m})\end{array}$ & $\begin{array}{l}\text { Bandpass } \\
(\mu \mathrm{m})\end{array}$ & $\begin{array}{l}\text { Sky Flux } \\
\text { Jy } / \operatorname{arcsec}^{2}\end{array}$ & $\begin{array}{l}\text { Continuum } \\
\text { Mags/beam }\end{array}$ & $\begin{array}{l}\text { Continuum } \\
\text { Jy/beam }\end{array}$ & $\begin{array}{l}\text { Line } \\
\mathrm{W} / \mathrm{m}^{2} / \text { beam }\end{array}$ & Filter \\
\hline 2.425 & 0.034 & $1(-4)$ & 17.1 & $7(-5)$ & $1(-18)$ & $\mathrm{H}_{2}$ Q-branch \\
\hline \multirow[t]{2}{*}{3.299} & 0.074 & $8(-2)$ & 14.7 & $4(-4)$ & $8(-18)$ & PAHs \\
\hline & & 0.2 & 14.0 & $7(-4)$ & $2(-17)$ & \\
\hline \multirow[t]{2}{*}{3.51} & 0.62 & 0.1 & 15.5 & $2(-4)$ & & Broad Band L \\
\hline & & 0.4 & 14.7 & $4(-4)$ & & \\
\hline \multirow[t]{2}{*}{3.82} & 0.60 & 0.3 & 14.8 & $3(-4)$ & & Broad Band L' \\
\hline & & 0.6 & 14.1 & $5(-4)$ & & \\
\hline \multirow[t]{2}{*}{4.051} & 0.054 & 0.4 & 12.9 & $2(-3)$ & $2(-17)$ & $\mathrm{H} \operatorname{Br} \alpha$ \\
\hline & & 0.7 & 12.2 & $3(-3)$ & $3(-17)$ & \\
\hline \multirow[t]{2}{*}{4.67} & 0.16 & 2 & 11.7 & $3(-3)$ & & Broad Band M \\
\hline & & 3 & 11.0 & $6(-3)$ & & \\
\hline
\end{tabular}

Sensitivities are $3 \sigma$ in 1 hour of on-source integration, assuming the signals of $5 \times 50.66^{\prime \prime}$ pixels are summed. For each filter the first line is the estimated winter-time sensitivity $(\mathrm{T}=213 \mathrm{~K})$ and the second-line in summer $(\mathrm{T}=243 \mathrm{~K})$ (except for $2.4 \mu \mathrm{m}$ where daytime measurements would not be sensible). Measured (extrapolated at M) sky fluxes at the Pole are used, and the 60-cm aperture SPIREX telescope assumed to have a $10 \%$ emissivity, a system throughput of $\sim 0.3$, and a detector with $1 \mathrm{e} / \mathrm{s}$ dark current, 100 e read noise and $\sim 80 \%$ qe. A maximum single integration time period of $600 \mathrm{~s}$ is assumed, which means the $\mathrm{H}_{2}$ filter is still detector noise limited; in principle another 0.8 mags. of sensitivity gain is possible through it.

- The star formation history of the Universe is being probed through deep, pencil-beam surveys, of which the Hubble Deep Field (HDF) is the most notable example. A southern field (the HDF-S) is shortly to be obtained. SPIREX/Abu will be capable of complementary deep $\mathrm{K}$ - and L-band imaging to the optical bands of the HDF-S. The 11' FOV of the instrument includes the three individual fields of the WFPC-2, STIS and NICMOS instruments. With 24 hours of on-source integration a detection threshold of 19.2 magnitudes through the $\mathrm{H}_{2} 2.4 \mu \mathrm{m}$ filter and 17.3 magnitudes through the L-band $3.5 \mu \mathrm{m}$ filter would be achieved. Based on the number counts in the HDF this would yield $\sim 40$ galaxies at $\mathrm{K}$ and $\sim 4$ at $\mathrm{L}$. These galaxies will typically have $<\mathrm{V}>=22.5, \mathrm{~V}-\mathrm{K}=3.5$ and a redshift $\mathrm{z}=0.6$. SPIREX/Abu would thus sample the upper end of the galaxy luminosity function, but with minimal uncertainties from extinction. However, it would also be sensitive to particularly red galaxies at high redshift; for instance an E/S0 galaxy at $\mathrm{z}=1.4$ has an (unreddened) $\mathrm{V}-\mathrm{L} \sim 10$ and so would be detected by $\mathrm{Abu}$ if 
$\mathrm{V}<27$. Moreover, statistics on the distribution of galaxies in the adjacent fields to the HDF-S would be simultaneously obtained.

The project serves as a demonstrator of the scientific potential of the Antarctic Plateau. While wide-field, deep, thermal infrared surveys are a particular niche this experiment can exploit, the work that a $60 \mathrm{~cm}$ telescope will do is limited. SPIREX/Abu is thus designed as a forerunner for an intermediate size infrared telescope, a project we call 'SPIRIT'.

\subsection{SPIRIT}

The 'South Pole Infrared Imaging Telescope' is envisaged as a $2 \mathrm{~m}$-class infrared optimised telescope capable of yielding near-diffraction limited images at $2.4 \mu \mathrm{m}$. It will be a wide-field telescope, instrumented with large format focal plane arrays, and operate primarily from $2-5 \mu \mathrm{m}$ and $10-30 \mu \mathrm{m}$. It will use a tiptilt mirror, which will recover most of the diffraction limit given that $\mathrm{r}_{0} \sim 36 \mathrm{~cm}$ at $2.4 \mu \mathrm{m}$. With a $0.25^{\prime \prime}$ pixel scale and $4^{\prime}$ field of view, scientific projects would emphasise deep surveys. The first programs would involve surveys of star forming regions at $3.8 \mu \mathrm{m}$ and $11.5 \mu \mathrm{m}$, to make a complete census of the embedded population of several such regions. Table 2 compares the sensitivity of SPIRIT to both the AAT and the new-generation 8-m telescopes like Gemini and the VLT. A $2.5 \mathrm{~m}$ telescope in Antarctica has comparable or better sensitivity than an 8-m telescope for both line and continuum imaging projects across the thermal infrared, with the additional advantage of having a wide field of view. It is clear that SPIRIT could yield unique new science. It would also provide a testbed facility for more ambitious telescopes in the future, capable of exploiting the full potential of the Antarctic Plateau for astronomy. SPIRIT seeks international partners to proceed, as part of the International Antarctic Observatory.

\section{Acknowledgements}

SPIREX/Abu has come about due to the dedicated efforts of a great many people working in extenuating and heroic circumstances. We especially wish to thank our colleagues at UNSW; Max Boccas, Mick Edgar, Andre Phillips, Antony Schinckel and Rodney Marks, who is wintering with the instrument. SPIREX/Abu is also the result of a happy and successful collaboration with several US institutions, and we are particularly grateful for the efforts of Bill Ball, Sean Casey, Darren Depoy, Al Fowler, Al Harper, Bob Loewenstein, Paul Martini, Fred Mrozak, Bob Pernic and Nigel Sharp.

\section{References}

Ashley, M.C.B., Burton, M.G., Storey, J.W.V., Lloyd, J.P., Bally, J., Briggs J.W., \& Harper, D.A. (1996): PASP, 108, 721

Burton, M.G. et al. (1994): Proc. Astron. Soc. Aust., 11, 127 
Harper, D.A. (1989): 'Astrophysics in Antarctica', Am. Inst. Phys. Conf. ser., 198, 123. Eds. D.J. Mullan, M.A. Pomerantz \& T. Stoner

Hereld, M. (1994): 'Infrared astronomy with arrays: the next generation', Ast. Ap. Sci. Lib., 190, 248. Ed I.S. McLean (Kluwer).

Marks, R.D., Vernin, J., Azouit, M., Briggs, J.W., Burton, M.G., Ashley, M.C.B. \& Manigault, J.F. (1996): A\&AS, 118, 385

Marks, R.D., Vernin, J., Azouit, M., Manigault, J.F. \& Clevelin, C. (1998): A\&A, in press.

Nguyen, H.T., Rauscher, B.J., Severson, S.A., Hereld, M., Harper, D.A., Loewenstein, R.F., Mrozek, F., \& Pernic, R.J. (1996): PASP, 108, 718

Smith, C.H. \& Harper, D.A. (1998): PASP, in press.

Storey, J.W.V. (1998): 'Astrophysics from Antarctica', PASPC, ASP meeting, Chicago, 30 June-2 July 1997, Eds G. Novak \& R.H. Landsberg, in press.

Strom, K.M., Strom, S.E., Edwards, S., Cabrit, S. \& Strutskie, M.F. (1989): AJ, 97, 1451 
Table 2. Sensitivity comparison between different Telescopes

\begin{tabular}{|c|c|c|c|c|c|}
\hline & & SPIRIT & $\mathrm{AAT}$ & Gemini / VLT & \\
\hline Diameter & & $2.5 \mathrm{~m}$ & $3.9 \mathrm{~m}$ & $8.0 \mathrm{~m}$ & \\
\hline Temperature & & $213 \mathrm{~K}$ & $288 \mathrm{~K}$ & $273 \mathrm{~K}$ & \\
\hline Emissivity & & 0.05 & 0.20 & 0.05 & \\
\hline Location & & South Pole & Siding Spring & Mauna Kea / & Atacama \\
\hline Wavelength & $\underline{\text { Resolution }}$ & & & & $\underline{\text { Filter }}$ \\
\hline$\overline{2.15 \mu \mathrm{m}}$ & $\mathrm{R}=7$ & & $\begin{array}{l}20.8 \text { mags } \\
3(-6) \mathrm{Jy}\end{array}$ & $\begin{array}{l}21.7 \text { mags } \\
1(-6) \mathrm{Jy}\end{array}$ & Broad Band, K-narrow \\
\hline 2.37 & 13 & $\begin{array}{l}21.9 \text { mags } \\
1(-6) \mathrm{Jy}\end{array}$ & & & Broad Band, K-dark \\
\hline 2.12 & 100 & & $\begin{array}{l}2(-19) \mathrm{W} / \mathrm{m}^{2} \\
1(-5) \mathrm{Jy}\end{array}$ & $\begin{array}{l}7(-20) \mathrm{W} / \mathrm{m}^{2} \\
5(-6) \mathrm{Jy}\end{array}$ & $\mathrm{H}_{2} \quad 1-0 \mathrm{~S}(1)$ \\
\hline 2.43 & 40 & $\begin{array}{l}7(-20) \mathrm{W} / \mathrm{m}^{2} \\
2(-6) \mathrm{Jy}\end{array}$ & & & $\begin{array}{l}\mathrm{H}_{2} 1-0 \text { Q-branch } \\
(\sim 3 \times 1-0 \mathrm{~S}(1) \text { intensity })\end{array}$ \\
\hline 3.30 & 45 & $\begin{array}{l}2(-18) \mathrm{W} / \mathrm{m}^{2} \\
7(-5) \mathrm{Jy}\end{array}$ & $\begin{array}{l}1(-17) \mathrm{W} / \mathrm{m}^{2} \\
6(-4) \mathrm{Jy}\end{array}$ & $\begin{array}{l}3(-18) \mathrm{W} / \mathrm{m}^{2} \\
1(-4) \mathrm{Jy}\end{array}$ & PAHs \\
\hline 3.60 & 46 & & $\begin{array}{l}14.8 \text { mags } \\
4(-4) \mathrm{Jy}\end{array}$ & $\begin{array}{l}16.1 \mathrm{mags} \\
1(-4) \mathrm{Jy}\end{array}$ & Narrow Band L \\
\hline 3.65 & 12 & $\begin{array}{l}17.3 \text { mags } \\
3(-5) \mathrm{Jy}\end{array}$ & & & Broad Band L \\
\hline 11.5 & 100 & & $\begin{array}{l}9.0 \mathrm{mags} \\
9(-3) \mathrm{Jy}\end{array}$ & $\begin{array}{l}10.2 \mathrm{mags} \\
3(-3) \mathrm{Jy}\end{array}$ & Narrow Band N \\
\hline 11.5 & 12 & $\begin{array}{l}11.5 \text { mags } \\
1(-3) \mathrm{Jy}\end{array}$ & & & Broad Band N \\
\hline 20.1 & 100 & $\begin{array}{l}8.3 \mathrm{mags} \\
5(-3) \mathrm{Jy}\end{array}$ & $\begin{array}{l}5.8 \text { mags } \\
5(-2) \mathrm{Jy}\end{array}$ & $\begin{array}{l}8.2 \text { mags } \\
5(-3) \mathrm{Jy}\end{array}$ & Narrow Band Q \\
\hline
\end{tabular}

Sensitivities are $3 \sigma$ per square arcsecond for one hour of on-source integration. The comparison is made for the optimum spectral bandpasses for Antarctic and mid-latitude sites, as specified above. Aside from site conditions, aperture size and telescope emissivity, other parameters are held constant betwen the systems, so that a direct comparison of performance is possible. A system throughput of 0.2 , dark current $1 \mathrm{e} / \mathrm{s}$, read noise 10 e and detector qe of $90 \%$ is assumed. In the thermal IR both the stability of the background level and the atmospheric transmission will determine whether these sensitivities can be reached in practice; the lower water vapour content at the Pole makes this site superior in this respect. 